\title{
Technology in quest of the holy grail
}

\author{
Ran D. Goldman ${ }^{1}$ \\ Received: 15 October 2020 / Accepted: 2 December 2020 \\ (c) Canadian Association of Emergency Physicians (CAEP)/ Association Canadienne de Médecine d'Urgence (ACMU) 2020
}

Keywords Pain management · Pediatrics · Technology

Pain in children is a complex experience comprising of sensory, cognitive, behavioural, and psychological components [1]. Painful procedures, such as vaccinations, intravenous injections, laceration repair, and dressing changes for burn wounds are common in the pediatric emergency department (PED). Painful experiences during these procedures often lead to anxiety for patients, which can cause fear, and greatly affect compliance with care and future procedures [2].

Acute pain management in children, especially during procedures, has been transformed since Swafford and Allen reported in 1968 that "Pediatric patients seldom need relief of pain after general surgery. They tolerate discomfort well" [3]. The 3P approach to pain management, a mix of psychological, physical and pharmacological strategies, is being taught and implemented in PEDs, pediatric wards and clinics [4]. As part of this approach, distraction is a simple and effective technique that directs children's attention away from noxious stimuli [5]. New technologies may help Emergency Medicine providers in enhancing comfort and reducing pain during procedures.

In this issue of CJEM, two Canadian groups evaluated the use of technology for management of pain and distress in PED intravenous (IV) insertion, albeit with lukewarm results. Osmanlliu et al. from Montreal [6] reported that Virtual Reality (VR) is feasible and acceptable among children in the PED and decreased distress and memory of pain, but did not reduce pain, compared to standard of care (SOC). Ali et al. from Edmonton reported that distraction of a robot playing with the child had a modest decrease of the child's distress, and increased parental satisfaction of

Ran D. Goldman

rgoldman@cw.bc.ca

1 The Innovative VR Pain Lab, Pediatric Research in Emergency Therapeutics (PRETx) Program, Division of Emergency Medicine, Department of Pediatrics, University of British Columbia, and the BC Children's Hospital, 4480 Oak St, Vancouver, BC, Canada pain management, yet it did not reduce pain, compared to SOC [7].

The multitude of technologies associated with distraction has yet to reach the holy grail of an "outchless ED". Take the promising VR technology for example. It does not interrupt the pain signals, but acts on pain perception and signaling through attention, emotion and concentration [8]. Objective functional magnetic resonance imaging (MRI) of healthy subjects using VR showed a greater than $50 \%$ reduction in pain-related brain activity in five areas of the brain [9]. Despite these findings, studies show conflicting results. Gold et al. reported VR to significantly reduce acute procedural pain and anxiety compared with SOC, [10] while our group from Vancouver recently found VR not to be significantly better than SOC for IV placement in the PED, [11] similar to Osmanlliu et al. [6].

There could be several explanations to this gap in findings related to pain. First, many PEDs in Canada are using advanced distraction techniques and employ child-life specialists in the ED, who can help children prepare and cope during procedures, so the SOC is setting a high bar to pass. Those new technologies may be better employed in nonPEM programs or EDs with limited distraction tools and abridged pediatric-trained personnel. Second, studying technology and devices is challenging. Studies are usually open-label, including those in this CJEM journal. With no blinding to the intervention, reporting may be biased, especially in face of subjective reporting of pain by children, parents and those conducting the procedures. Methodology needs to evolve to enhance blinding, which is a challenge with VR goggles. One idea may be to use a sham procedure. Consider allowing all children to use VR, but some children will have a needle procedure and others a sham procedure with no needle penetration into the skin. Data from the first group can be used.

The third challenge is the measurement of pain, anxiety or distress. While several scales have been validated 
in children, majority were not specifically validated in the acute care PED setting, or for the procedures investigated. This may result in a meaningful bias in reporting, especially when children of all ages (such as in this CJEM issue studies; 6-11 and 7-17 years ) are included in the same trial. In future reports, it may be prudent to limit assessment for a narrow age group.

Finally, there may be a "novelty bias". Children seem to have 'fun' and 'excitement' around the technology that they are introduced to in the PED during those trials, which may facilitate their abridged anxiety and distress, as well as enhanced satisfaction. One may wonder if those advances will fade as devices and technologies (such as VR) appear in more houses as the gaming industry stretches inexpensive technologies to our homes.

With the advent of affordable consumer-grade equipment and applications, and a very good safety profile, technologies such as VR, robot-assisted play-therapy and others may serve as tools clinicians can dispose to enhance the "ED Experience". Age-and-indication-appropriate studies and scientifically-sound methodology will help hone in on the groups of children that will appreciate technologies during procedures in PEDs and eventually will also.... enjoy alleviated pain.

\section{References}

1. Morley S, Williams A. New developments in the psychological management of chronic pain. Can J Psychiatry. 2015;60(4):16875. https://doi.org/10.1177/070674371506000403.
2. Oliveira NCAC, Gaspardo CM, Linhares MBM. Pain and distress outcomes in infants and children: a systematic review. Braz J Med Biol Res. 2017;50(7):e5984. https://doi.org/10.1590/1414-431X2 0175984.

3. Swafford LI, Allen D. Pain relief in the pediatric patient. Med Clin North Am. 1968;52:131-5.

4. Pope N, Tallon M, McConigley R, Wilson S. The experiences of acute non-surgical pain of children who present to a healthcare facility for treatment: a systematic review protocol. JBI Database Syst Rev Implement Rep. 2015;13(10):12-20. https://doi. org/10.11124/jbisrir-2015-2466.

5. Koller D, Goldman RD. Distraction techniques for children undergoing procedures: a critical review of pediatric research. J Pediatr Nurs. 2012;27(6):652-81. https://doi.org/10.1016/j. pedn.2011.08.001 (Epub 2011 Oct 13).

6. Osmanlliu E, Trottier ED, Bailey B, et al. Distraction in the emergency department using virtual reality for INtravenous procedures in Children to Improve comfort (DEVINCI): a pilot pragmatic randomized controlled trial. Can J Emerg Med. 2020. https://doi. org/10.1007/s43678-020-00006-6.

7. Ali S, Manaloor R, Ma K, et al. A randomized trial of robot-based distraction to reduce children's distress and pain during intravenous insertion in the emergency department. Can J Emerg Med. 2020. https://doi.org/10.1007/s43678-020-00023-5.

8. Arane K, Behboudi A, Goldman RD. Virtual reality for pain and anxiety management in children. Can Fam Physician. 2017;63(12):932-4.

9. Hoffman HG, Richards TL, Coda B, Bills AR, Blough D, Richards AL, et al. Modulation of thermal pain-related brain activity with virtual reality: evidence from fMRI. NeuroReport. 2004;25(7):2013-4.

10. Gold JI, Mahrer NE. Is Virtual Reality Ready for Prime Time in the Medical Space? A Randomized Control Trial of Pediatric Virtual Reality for Acute Procedural Pain Management. J Pediatr Psychol. 2018;43(3):266-75.

11. Goldman RD, Behboudi A. Virtual reality for intravenous placement in the emergency department-a randomized controlled trial. Eur J Pediatr. 2020. doi: https://doi.org/10.1007/s00431-02003771-9. Epub ahead of print. 